\title{
Comparison of treatment outcomes between squamous cell carcinoma and adenocarcinoma of cervix after definitive radiotherapy or concurrent chemoradiotherapy
}

\author{
Ke $\mathrm{Hu}^{\dagger}$, Weiping Wang ${ }^{\dagger}$, Xiaoliang Liu, Qingyu Meng and Fuquan Zhang*i(
}

\begin{abstract}
Background: Concurrent chemoradiotherapy (CCRT) is effective in the treatment of locally advanced cervical squamous cell carcinoma (SCC). However, whether treatment outcomes of cervical adenocarcinoma are equivalent to SCC after CCRT has been a topic of debate.

Methods: Medical records of cervical cancer patients treated with definitive radiotherapy or CCRT in our institute from January 2011 to December 2014 were reviewed. Patients were treated with intensity modulated radiation therapy combined with intracavitary brachytherapy. Weekly cisplatin was the first line regimen of concurrent chemotherapy. The treatment outcomes of patients with SCC and adenocarcinoma were compared with a multivariate Cox regression model, and log-rank method before and after propensity score matching (1:1).

Results: A total of 815 patients with stage IB-IVA cervical cancer were included, with 744 patients in the SCC group and 71 patients in adenocarcinoma group. The median follow-up period was 36.2 months (range, 1.0-76.2 months). The 3-year overall survival (OS), disease-free survival (DFS), pelvic control and distant control rates of patients in the SCC group and adenocarcinoma group were 85.2 and $75.4 \%(p=0.005), 77.5$ and $57.3 \%(p<0.001), 89.0$ and $74.0 \%(p=0.001)$ and 86.0 and 74.4\% ( $p=0.011)$, respectively. After multivariate analysis, histology was an independent factor of OS $(p=0.003)$, DFS $(p<0.001)$, pelvic control $(p=0.002)$ and distant control $(p=0.003)$. With propensity score matching, 71 pairs of patients were selected. After matching, the OS $(p=0.017)$, DFS $(p=0.001)$, pelvic control $(p=0.015)$ and distant control $(p=0.009)$ of patients with adenocarcinoma were poorer than those of patients with SCC. In subgroup analysis, patients with adenocarcinoma had significantly worse OS and DFS compared with patients with SCC, regardless of treatment with radiotherapy alone or CCRT.
\end{abstract}

Conclusion: The present study demonstrated that patients with adenocarcinoma of the cervix had poorer OS and DFS than patients with SCC, regardless of treatment with radiotherapy alone or CCRT. New treatment approaches should be considered for cervical adenocarcinoma.

Keywords: Adenocarcinoma, Squamous cell carcinoma, Cervical cancer, Radiotherapy, Concurrent chemoradiotherapy

\footnotetext{
* Correspondence: zhangfuquan3@sina.com

${ }^{\dagger} \mathrm{Ke} \mathrm{Hu}$ and Weiping Wang contributed equally to this work.

Department of Radiation Oncology, Peking Union Medical College Hospital,

Chinese Academy of Medical Sciences and Peking Union Medical College,

No.1 Shuaifuyuan Wangfujing, Dongcheng District, Beijing 100730, China
}

(c) The Author(s). 2018 Open Access This article is distributed under the terms of the Creative Commons Attribution 4.0 International License (http://creativecommons.org/licenses/by/4.0/), which permits unrestricted use, distribution, and

reproduction in any medium, provided you give appropriate credit to the original author(s) and the source, provide a link to the Creative Commons license, and indicate if changes were made. The Creative Commons Public Domain Dedication waiver (http://creativecommons.org/publicdomain/zero/1.0/) applies to the data made available in this article, unless otherwise stated. 


\section{Background}

Cervical cancer is one of the most common gynaecological cancers in women. It was estimated that there were 13,240 new cases and 4170 deaths in United States in 2018 [1]. Squamous cell carcinoma (SCC) accounts for approximately $70 \%$ of all cervical cancer and adenocarcinoma (AC) accounts for approximately 20\% [2]. With screening and human papilloma virus vaccines, the incidence of cervical cancer had significantly decreased in recent years in developed countries [2-4]. However, incidence of $\mathrm{AC}$ of the cervix has increased in past decades. The age-adjusted AC incidence rates increased 0.5 to $3 \%$ per annum in Europe [5]. The probable reason is that, compared with cervical SCC, cervical cytologic screening methods are less effective for cervical AC [6].

At present, there is no difference in treatments between SCC and AC of the cervix. However, clinical characteristics and prognosis of cervical AC differ from SCC. Patients with $\mathrm{AC}$ have been reported to be younger, more often white, more commonly diagnosed while in the early stages and more likely to have metastatic lymph nodes (MLNs) than patients with SCC [7, 8]. Whether patients with different histological subtype have different survival outcomes remains a topic of debate. In some previous studies, for early stage cervical cancer patients treated with hysterectomy and lymphadenectomy, cervical AC exhibited equivalent survival to SCC [9-11]. In some other studies, after radical hysterectomy and lymph node dissection, AC was associated with a poor survival, compared with SCC [12-14].

Currently, the standard treatment approach for locally advanced cervical cancer patients is definitive concurrent chemoradiotherapy (CCRT), regardless of the histological subtype of the disease. Studies on the prognostic significance of AC in cervical cancer patients treated with CCRT are limited, and the results are conflicting [7, 15-18]. In a study from Japan, patients with AC or adenosquamous carcinoma (ASC) of the cervix experienced worse overall survival (OS) $(p=0.004)$ and progressionfree survival (PFS) $(p=0.002)$ than patients with SCC of the cervix after definitive radiotherapy. AC/ASC was also an independent prognostic factor of PFS in multivariate analysis $(p=0.031)$ [15]. A study based on the Korea National Cancer Incidence Database showed that the survival of patients with cervical AC improved after the introduction of concurrent chemotherapy. However, AC was still associated with worse OS compared with SCC for patients treated with CCRT $(p=0.003)$ [17]. Rose et al. retrospectively analysed 1671 cervical cancer patients (1489 patients with SCC and 182 patients with AC/ASC) in five Gynaecologic Oncology Group (GOG) trials, and found that when treated with radiotherapy alone, AC/ASC was associated with poor OS $(p=0.0449)$. However, when patients were treated with cisplatin-based CCRT, the OS $(p=0.459)$ and PFS $(p=0.315)$ were similar among patients with SCC and AC/ASC [7]. In the study of Katanyoo et al., 141 patients with $\mathrm{AC}$ were matched with 282 patients with SCC. After radiotherapy/CCRT, the complete response $(\mathrm{CR})$ rates in patients with $\mathrm{AC} / \mathrm{ASC}$ and SCC were 86.5 and $94.7 \%$, respectively $(p=0.004)$. However, the 5 -year OS was similar, $59.9 \%$ in patients with AC and $61.7 \%$ in patients with SCC $(p=0.191)$ [18].

In the present study, we reviewed cervical patients treated with radiotherapy or CCRT in our institute and analysed the prognostic significance of AC.

\section{Methods \\ Patients}

Medical records of cervical cancer patients treated with definitive radiotherapy or CCRT in our institute from January 2011 to December 2014 were reviewed. The inclusive criteria included the following: biopsy confirmed cervical AC or SCC; the International Federation of Gynaecology and Obstetrics (FIGO) stage IB-IVA; no evidence of distant metastases; treated with definitive radiotherapy or CCRT. Patients with all histological types except SCC and AC, including ASC, undifferentiated carcinoma, neuroendocrine carcinoma, sarcoma, lymphoma and other scarce histology, were excluded from this study.

\section{Treatment}

Patients were treated with definitive intensity modulated radiation therapy (IMRT) and intracavitary brachytherapy (ICBT). The clinical target volume (CTV) and gross tumour volume (GTVnd) were contoured on CT simulation slices. The CTV included the cervix, uterus, parametrium, upper part of the vagina and pelvic lymph node regions. For patients with para-aortic MLNs, or with high risk of para-aortic lymph node failure, the para-aortic lymph node region was also included in the CTV $[19,20]$. GTVnd covered the regional MLNs. A margin of 8 to $10 \mathrm{~mm}$ was added to the CTV in all directions and an additional 5 to $10 \mathrm{~mm}$ margin to the uterus and cervix to create the planning target volume (PTV). A 5-mm margin was given to GTVnd to generate planning gross tumour volume (PGTVnd). A dose of 50.4 Gy in 28 fractions was prescribed to the PCTV with IMRT, and the PGTVnd were simultaneously boosted to 59-61 Gy. For patients with stage IIIB disease, an additional dose of $10 \mathrm{~Gy}$ in 5 fractions was delivered to the parametrium in our institute. Daily megavoltage computed tomography (MVCT) or weekly cone-beam CT/CT-on-rail were used for image guidance. 
ICBT was delivered with iridium-192, with 30 to 36 Gy in 5 to 7 fractions prescribed to point A. The details of radiotherapy were described previously [21, 22].

The first line regimen of concurrent chemotherapy was cisplatin $40 \mathrm{mg} / \mathrm{m}^{2}$ weekly. Patients with renal dysfunction underwent paclitaxel weekly. Part of patients with AC of the cervix received paclitaxel or paclitaxel plus cisplatin.

\section{Patient follow-up}

Patients had a follow-up evaluation 1 month after treatment. After that, patients received follow-up examinations every 3 months in the first 2 years, every 6 months in years 3 to 5 and once a year thereafter. SCC antigen, chest/abdomen CT and pelvic MRI were conducted regularly in follow-up. Positron emission tomography (PET)-CT was used for patients with suspected recurrence or metastasis.

\section{Statistics}

The endpoints included OS, disease-free survival (DFS), pelvic control and distant control. The baseline characteristics between patients in the SCC and AC groups were compared with chi-squared $(x 2)$ or continuity correction tests, as appropriate. Univariate analysis and multivariate analysis were performed with a Cox proportional hazard regression model. As the baseline characteristics were significantly different between two groups, propensity score matching was performed with a ratio of 1:1. The matching covariate included para-aortic MLNs, pelvic MLNs and concurrent chemotherapy. OS, DFS, pelvic control and distant control were estimated using the Kaplan-Meier method and compared between the SCC and AC groups using the log-rank method before and after matching. In subgroup analysis, the Cox regression model or log-rank methods were used to compare the survival between subgroups. Statistical analyses were performed with SPSS (version 22.0; SPSS, Inc., Chicago, IL, USA). Two-sided $p<0.05$ was considered statistically significant.

\section{Results}

Between January 2011 and December 2014, there were 833 patients with stage IB-IVA cervical cancer treated with definitive radiotherapy or CCRT in our institute. Nine patients with ASC, seven patients with undifferentiated carcinoma, and two patients with neuroendocrine carcinoma were excluded. Finally, 815 were patients enrolled in this study. Of them, there were 744 patients (91.3\%) with SCC and 71 patients (8.7\%) with AC. The detailed characteristics of patients in the SCC and AC groups are shown in Table 1. More patients in the AC group had para-aortic MLNs $(5.9 \%$ in SCC group and $14.1 \%$ in AC group, $p=0.008$ ), and more patients in the SCC group had pelvic MLNs $29.2 \%$ in SCC group and $19.7 \%$ in AC group, $p=0.091)$. The other variables, including age, FIGO stage, primary tumour size, common iliac MLNs, number of pelvic MLNs and CCRT were similar between two groups.

\section{Survivals of patients in SCC and AC groups}

The median follow-up period was 36.2 months (range, 1.0-76.2 months) for the whole cohort, with 37.3 months (range, 1.0-76.2 months) in the SCC group and 28.8 months (range, 5.1-68.8 months) in the AC group ( $p<$ 0.001). The 3-year OS, DFS, pelvic control and distant control rates in the SCC and AC groups were 85.2 and $75.4 \%(p=0.005), 77.5$ and $57.3 \%(p<0.001), 89.0$ and $74.0 \%(p=0.001)$ and 86.0 and $74.4 \%(p=0.011)$, respectively. The OS, DFS, pelvic control and distant control curves of patients treated with SCC and AC are shown in Fig. 1.

\section{Univariate and multivariate analysis}

After univariate analysis (Table 2), histology was a significant factor of OS $(p=0.006)$, DFS $(p<0.001)$, pelvic control $(p=0.002)$ and distant control $(p=0.013)$. Significant factors in univariate analysis were analysed with multivariate analysis. As shown in Table 3, histology remained significant in predicting OS (hazard ratio, HR 2.21, 95\% confidence interval, CI 1.31-3.74, $p=0.003$ ), DFS (HR 2.37, 95\% CI 1.57-3.56, $p<0.001$ ), pelvic control (HR 2.40, 95\% CI 1.36-4.22, $p=0.002$ ) and distant control (HR 2.27, 95\% CI 1.31-3.92, $p=0.003)$ after multivariate analysis.

\section{Propensity score matching}

To balance the basic characteristics, 71 cervical patients with AC were matched with 71 patients with SCC. The basic characteristics were similar between two groups (Table 1).

After matching, the 3-year OS, DFS, pelvic control and distant control rates of patients in the SCC and AC groups were 86.4 and $75.4 \%$ ( $p=0.017), 82.5$ and $57.3 \%$ $(p=0.001), 91.2$ and $74.0 \%(p=0.015)$ and 91.1 and $74.4 \%(p=0.009)$, respectively.

\section{Subgroup analysis}

In 142 patients treated with definitive radiotherapy alone, 11 patients had AC and 131 patients had SCC. The OS (HR 2.64, 95\% CI 1.01-6.87, $p=0.047$ ), DFS (HR 3.46, 95\% CI 1.60-7.46, $p=0.002$ ) and distant control (HR 3.22, 95\% CI 1.09-9.51, $p=0.035$ ) of patients with AC were worse than patients with SCC. The pelvic control (HR 2.12, 95\% CI 0.62-7.20, $p=0.229$ ) was not 
Table 1 Characteristics of cervical cancer patients with SCC and AC before and after matching

\begin{tabular}{|c|c|c|c|c|c|c|}
\hline \multirow[t]{2}{*}{ Characteristics } & \multicolumn{3}{|c|}{ Before matching } & \multicolumn{3}{|c|}{ After matching } \\
\hline & $\operatorname{SCC}(n=744)$ & $\mathrm{AC}(n=71)$ & $p$ & $\operatorname{SCC}(n=71)$ & $\mathrm{AC}(n=71)$ & $\mathrm{p}$ \\
\hline \multicolumn{7}{|l|}{ Age (ys) } \\
\hline$<65$ & $663(89.1 \%)$ & $64(90.1 \%)$ & 0.790 & $66(93.0 \%)$ & $64(90.1 \%)$ & 0.546 \\
\hline$\geq 65$ & $81(10.9 \%)$ & 7 (9.9\%) & & $5(7.0 \%)$ & 7 (9.9\%) & \\
\hline \multicolumn{7}{|l|}{ FIGO stage } \\
\hline Stage I & $92(12.4 \%)$ & 7 (9.9\%) & 0.359 & $3(4.2 \%)$ & 7 (9.9\%) & 0.403 \\
\hline Stage II & 505 (67.9\%) & $54(76.1 \%)$ & & $56(78.9 \%)$ & $54(76.1 \%)$ & \\
\hline Stage III-IVA & $147(19.8 \%)$ & $10(14.1 \%)$ & & $12(16.9 \%)$ & $10(14.1 \%)$ & \\
\hline \multicolumn{7}{|c|}{ Primary tumour size } \\
\hline$<4 \mathrm{~cm}$ & 287 (38.6\%) & 29 (40.8\%) & 0.708 & $28(39.4 \%)$ & $29(40.8 \%)$ & 0.864 \\
\hline$\geq 4 \mathrm{~cm}$ & $457(61.4 \%)$ & $42(59.2 \%)$ & & $43(60.6 \%)$ & $42(59.2 \%)$ & \\
\hline \multicolumn{7}{|c|}{ Para-aortic MLNs } \\
\hline Yes & $44(5.9 \%)$ & $10(14.1 \%)$ & 0.008 & $10(14.1 \%)$ & $10(14.1 \%)$ & 1.000 \\
\hline No & $700(94.1 \%)$ & 61 (85.9\%) & & $61(85.9 \%)$ & $61(85.9 \%)$ & \\
\hline \multicolumn{7}{|l|}{ Pelvic MLNs } \\
\hline Yes & $217(29.2 \%)$ & $14(19.7 \%)$ & 0.091 & $14(19.7 \%)$ & $14(19.7 \%)$ & 1.000 \\
\hline No & $527(70.8 \%)$ & $57(80.3 \%)$ & & $57(80.3 \%)$ & $57(80.3 \%)$ & \\
\hline \multicolumn{7}{|c|}{ Common iliac MLNs } \\
\hline Yes & $56(7.5 \%)$ & $6(8.5 \%)$ & 0.779 & 7 (9.9\%) & $6(8.5 \%)$ & 0.771 \\
\hline No & $688(92.5 \%)$ & 65 (91.5\%) & & $64(90.1 \%)$ & 65 (91.5\%) & \\
\hline \multicolumn{7}{|c|}{ Number of pelvic MLNs } \\
\hline $0-3$ & 705 (94.8\%) & 68 (95.8\%) & 0.711 & $65(91.5 \%)$ & 68 (95.8\%) & 0.491 \\
\hline$\geq 4$ & $39(5.2 \%)$ & $3(4.2 \%)$ & & $6(8.5 \%)$ & $3(4.2 \%)$ & \\
\hline \multicolumn{7}{|c|}{ Concurrent chemotherapy } \\
\hline Yes & $613(82.4 \%)$ & $60(84.5 \%)$ & 0.654 & $56(78.9 \%)$ & $60(84.5 \%)$ & 0.385 \\
\hline No & $131(17.6 \%)$ & $11(15.5 \%)$ & & $15(21.1 \%)$ & $11(15.5 \%)$ & \\
\hline
\end{tabular}

AC adenocarcinoma, FIGO the International Federation of Gynaecology and Obstetrics, MLNs metastatic lymph nodes, SCC squamous cell carcinoma

significantly different. As for 673 patients treated with CCRT (60 patients with AC and 613 patients with SCC), AC was associated with worse OS (HR 1.85, 95\% CI $1.03-3.31, p=0.039$ ), DFS (HR 1.97, 95\% CI 1.25-3.09, $p=0.003$ ) and pelvic control (HR 2.42, 95\% CI 1.33$4.41, p=0.004)$. The distant control was not significantly different between the two groups (HR 1.73, 95\% CI $0.95-3.18, p=0.076$ ).

Of the 815 patients, 96 patients experienced pelvic failure. In the other 719 patients (55 patients with AC and 664 patients with SCC) without pelvic recurrence, patients with AC also had poorer DFS (HR 2.13, 95\% CI 1.21-3.74, $p=0.009$ ) and distant control (HR 1.98, 95\% CI 1.08-3.64, $p=0.027)$ than those with SCC. The OS (HR 1.52, 95\% CI 0.66-3.54, $p=0.329$ ) was similar in these two groups.

In 71 patients with $\mathrm{AC}$ of the cervix, 60 patients were treated with CCRT, with 46 patients receiving cisplatin, 6 patients receiving paclitaxel and 8 patients receiving paclitaxel plus cisplatin. The 3-year OS,
DFS, pelvic control and distant control rates of AC patients treated cisplatin and paclitaxel/paclitaxel plus cisplatin were 78.5 and $84.6 \%(p=0.819), 54.0$ and 85.1\% $(p=0.163), 72.1$ and $85.1 \%(p=0.149)$ and 71.8 and $85.1 \%(p=0.425)$.

\section{Discussion}

In the present study, we found that patients with $\mathrm{AC}$ had worse survival, compared with patients with SCC. In the SCC and AC groups, the 3-year OS rates were 85.4 and $75.4 \%$, respectively $(p=0.005)$, and the 3 -year DFS was 77.5 and $57.3 \%$, respectively $(p<0.001)$. Considering that there were more patients in the $\mathrm{AC}$ group with para-aortic MLNs, we conducted multivariate analysis and propensity score matching to verify the results. With multivariate analysis, AC was an independent prognostic factor of OS $(p=0.003)$ and DFS $(p<0.001)$. After propensity score matching at a ratio of 1:1, the 3-year OS rates were 86.4 and $75.4 \%(p=0.017)$ in the SCC and $\mathrm{AC}$ groups, and the 3 -year DFS rates were 82.5 and 

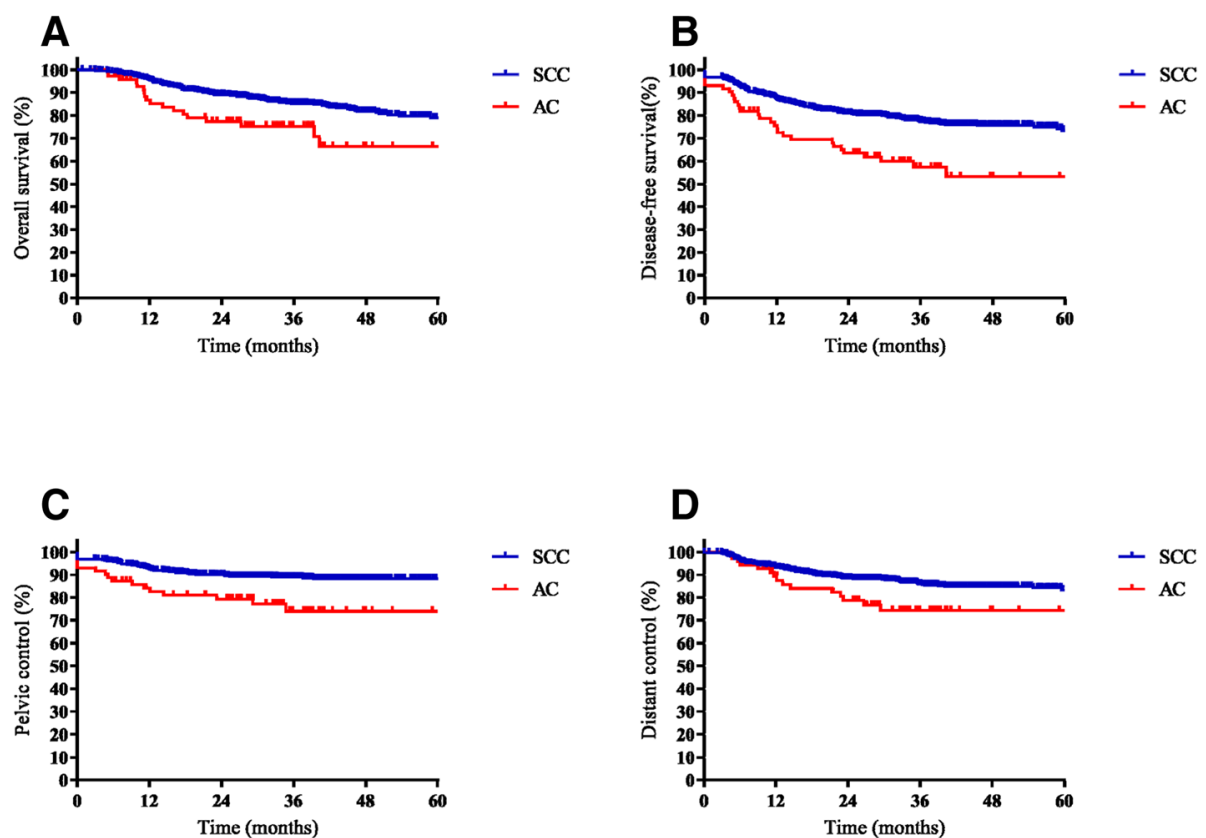

Fig. 1 The overall survival (OS, a), disease-free survival (DFS, b), pelvic control (c) and distant control (d) of patients with squamous cell carcinoma $(\mathrm{SCC})$ and adenocarcinoma $(\mathrm{AC})$ of the cervix

$57.3 \%(p=0.001)$. Patients in the AC group had significantly worse survival.

A previous study suggested that the survival of patients with cervical AC/ASC was poor when treated with radiotherapy alone. With CCRT, the survival was similar between AC/ASC and SCC groups [7]. In our study, regardless of treatment with radiotherapy or CCRT, OS and DFS were significantly different between two groups. It should be noted that the HR values of DFS were 3.22 for patients receiving radiotherapy alone, and 1.97 for patients treated with CCRT. This may indicate that the survival difference between AC and SCC of the cervix became smaller with the introduction of concurrent chemotherapy.
The poor radio-sensitivity of cervical $\mathrm{AC}$ is one of the causes of worse survival of AC. It has been reported that patients with $\mathrm{AC}$ had poorer complete response $(C R)$ and local control rates and required a longer time to achieve $\mathrm{CR}$ compared with patients with SCC after definitive radiotherapy or CCRT [15, 16 , 18]. Similarly, patients with AC experienced more local failure in our study. We also found that patients with AC were more likely to have distant failure. This was significant in the comparison with log-rank method before $(p=0.011)$ and after matching $(p=0.009)$. In multivariate analysis, $\mathrm{AC}$ was an independent factor of distant control $(p=0.003)$. Even in patients with pelvic control, patients with

Table 2 Results of univariate analysis for OS, DFS, pelvic control and distant control

\begin{tabular}{|c|c|c|c|c|c|c|c|c|}
\hline \multirow[t]{2}{*}{ Variables } & \multicolumn{2}{|l|}{ OS } & \multicolumn{2}{|l|}{ DFS } & \multicolumn{2}{|l|}{ Pelvic control } & \multicolumn{2}{|l|}{ Distant control } \\
\hline & $\mathrm{HR}(95 \% \mathrm{Cl})$ & $p$ & HR $(95 \%$ Cl) & $\mathrm{p}$ & $\mathrm{HR}(95 \% \mathrm{Cl})$ & $p$ & $\mathrm{HR}(95 \% \mathrm{Cl})$ & $p$ \\
\hline Age (<65 vs $\geq 65)$ & $2.01(1.29-3.13)$ & 0.002 & $1.38(0.92-2.07)$ & 0.124 & $0.99(0.51-1.90)$ & 0.968 & $1.20(0.69-2.11)$ & 0.516 \\
\hline Histology (SCC vs AC) & $2.01(1.22-3.31)$ & 0.006 & $2.21(1.49-3.25)$ & $<0.001$ & $2.33(1.36-3.99)$ & 0.002 & $1.96(1.15-3.32)$ & 0.013 \\
\hline FIGO stage (I, II and III-IVA) & $2.24(1.65-3.06)$ & $<0.001$ & $1.96(1.52-2.52)$ & $<0.001$ & $1.87(1.31-2.69)$ & 0.001 & $1.76(1.26-2.45)$ & 0.001 \\
\hline Tumour size $(<4 \mathrm{~cm}$ vs $\geq 4 \mathrm{~cm})$ & $2.74(1.80-4.17)$ & $<0.001$ & $2.43(1.74-3.39)$ & $<0.001$ & $2.60(1.59-4.26)$ & $<0.001$ & $2.20(1.44-3.36)$ & $<0.001$ \\
\hline Para-aortic MLNs (No vs Yes) & $6.09(4.05-9.15)$ & $<0.001$ & $5.10(3.55-7.34)$ & $<0.001$ & $6.66(4.18-10.60)$ & $<0.001$ & $3.05(1.77-5.26)$ & $<0.001$ \\
\hline Pelvic MLNs (No vs Yes) & $3.21(2.28-4.50)$ & $<0.001$ & $2.90(2.20-3.84)$ & $<0.001$ & $3.23(2.16-4.82)$ & $<0.001$ & $2.63(1.82-3.79)$ & $<0.001$ \\
\hline Common iliac MLNs (No vs Yes) & $5.68(3.83-8.43)$ & $<0.001$ & $4.17(2.91-5.98)$ & $<0.001$ & $3.55(2.12-5.93)$ & $<0.001$ & $4.20(2.64-6.70)$ & $<0.001$ \\
\hline Number of pelvic MLNs (continuous) & $1.29(1.24-1.35)$ & $<0.001$ & $1.27(1.22-1.32)$ & $<0.001$ & $1.25(1.19-1.32)$ & $<0.001$ & $1.22(1.15-1.28)$ & $<0.001$ \\
\hline Concurrent chemotherapy (No vs Yes) & $0.67(0.45-0.99)$ & 0.046 & $0.71(0.51-0.99)$ & 0.044 & $0.72(0.44-1.16)$ & 0.178 & $0.83(0.53-1.32)$ & 0.433 \\
\hline
\end{tabular}

AC adenocarcinoma, Cl confidence interval, FIGO the International Federation of Gynaecology and Obstetrics, HR hazard ratio, MLNs metastatic lymph nodes, SCC squamous cell carcinoma 
Table 3 Results of multivariate analysis for OS, DFS, pelvic control and distant control

\begin{tabular}{|c|c|c|c|c|c|c|c|c|}
\hline \multirow[t]{2}{*}{ Variables } & \multicolumn{2}{|l|}{ OS } & \multicolumn{2}{|l|}{ DFS } & \multicolumn{2}{|l|}{ Pelvic control } & \multicolumn{2}{|l|}{ Distant control } \\
\hline & $\mathrm{HR}$ & $p$ & $\mathrm{HR}$ & $P$ & $\mathrm{HR}$ & $P$ & $\mathrm{HR}$ & $P$ \\
\hline Age $(<65$ vs $\geq 65)$ & $2.07(1.27-3.36)$ & 0.004 & & & & & & \\
\hline Histology (SCC vs AC) & $2.21(1.31-3.74)$ & 0.003 & $2.37(1.57-3.56)$ & $<0.001$ & $2.40(1.36-4.22)$ & 0.002 & $2.27(1.31-3.92)$ & 0.003 \\
\hline FIGO stage (I, II and III-IVA) & $1.84(1.33-2.56)$ & $<0.001$ & $1.56(1.20-2.04)$ & 0.001 & $1.41(0.96-2.06)$ & 0.079 & $1.46(1.03-2.07)$ & 0.036 \\
\hline Tumour size $(<4 \mathrm{~cm}$ vs $\geq 4 \mathrm{~cm})$ & $2.00(1.28-3.14)$ & 0.002 & $1.80(1.27-2.54)$ & 0.001 & $1.81(1.08-3.03)$ & 0.024 & $1.70(1.10-2.65)$ & 0.018 \\
\hline Para-aortic MLNs (No vs Yes) & $1.28(0.69-2.40)$ & 0.434 & $1.40(0.85-2.33)$ & 0.188 & $2.42(1.25-4.68)$ & 0.008 & $0.73(0.35-1.56)$ & 0.420 \\
\hline Pelvic MLNs (No vs Yes) & $1.43(0.91-2.24)$ & 0.125 & $1.54(1.08-2.22)$ & 0.018 & $1.62(0.97-2.71)$ & 0.065 & $1.49(0.93-2.38)$ & 0.097 \\
\hline Common iliac MLNs (No vs Yes) & $1.30(0.72-2.35)$ & 0.392 & $1.08(0.65-1.78)$ & 0.773 & $0.69(0.34-1.40)$ & 0.304 & $1.67(0.88-3.17)$ & 0.119 \\
\hline Number of pelvic MLNs (continuous) & $1.16(1.07-1.26)$ & 0.001 & $1.14(1.06-1.23)$ & 0.001 & $1.13(1.03-1.25)$ & 0.009 & $1.13(1.02-1.25)$ & 0.018 \\
\hline Concurrent chemotherapy (No vs Yes) & $0.85(0.55-1.29)$ & 0.440 & $0.72(0.51-1.01)$ & 0.059 & & & & \\
\hline
\end{tabular}

AC adenocarcinoma, $\mathrm{Cl}$ confidence interval, FIGO the International Federation of Gynaecology and Obstetrics, HR hazard ratio, MLNs metastatic lymph nodes, SCC squamous cell carcinoma

AC still had poorer distant control $(p=0.027)$ and DFS $(p=0.009)$ than those with SCC. This indicated that poor radio-sensitivity was not the only the cause of worse survival of AC patients. Patients with AC also had a higher risk of distant recurrence after radiotherapy or CCRT.

Considering the poor survival of patients with AC of the cervix, we may need more effective protocols for these patients. One strategy is neoadjuvant or adjuvant chemotherapy. In a clinical trial from China, 880 patients with FIGO stage IIB-IVA cervical AC were randomised to receive either CCRT or CCRT with one cycle of neoadjuvant chemotherapy and two cycles of consolidation chemotherapy. The regimen for both neoadjuvant and consolidation chemotherapy were paclitaxel plus cisplatin. After a median follow-up period of 60 months, patients in the CCRT combined with neoadjuvant and adjuvant chemotherapy group experienced better DFS $(p<0.05)$, cumulative survival $(<0.05)$ and local control $(p<0.05)$. These results suggest that incorporating neoadjuvant and adjuvant chemotherapy to CCRT is a promising approach to improve the survival of patients with cervical AC [23]. Currently, cisplatin is the most favourable drug for concurrent chemotherapy. It is effective in the treatment of SCC of cervix. However, cisplatin may not be appropriate for patients with AC. Some studies have indicated that paclitaxel was an active agent for AC of the cervix [24, 25]. Huang et al. reported that, in cervical AC/ASC patients with advanced stage or MLNs, the 5-year relapse-free survival rates for patients treated with radiotherapy alone (45 patients), cisplatin-based CCRT (36 patients) and paclitaxel-based CCRT (13 patients) were $41.7,41.7$, and $53.8 \%$ ( $p=$ 0.611) [26]. In the present study, of the $60 \mathrm{AC}$ patients treated with CCRT, 14 patients received paclitaxel/paclitaxel plus cisplatin. In subgroup analysis, the 3-year OS, DFS, pelvic control and distant control rates of AC patients treated cisplatin and paclitaxel-based regimens were 78.5 and $84.6 \%(p=0.819), 54.0$ and $85.1 \%(p=$ $0.163), 72.1$ and $85.1 \%(p=0.149)$ and 71.8 and $85.1 \%$ $(p=0.425)$, respectively. Paclitaxel trended toward improved survival, although the differences were not significant. These suggested the efficacy of paclitaxel in concurrent setting.

\section{Conclusion}

The present study demonstrated that patients with AC of the cervix had poorer OS and DFS than patients with SCC, regardless of treatment with radiotherapy alone or CCRT. New treatment approaches should be considered for cervical AC.

\section{Abbreviations}

AC: Adenocarcinoma; ASC: Adenosquamous carcinoma; CCRT: Concurrent chemoradiotherapy; Cl: Confidence interval; CR: Complete response; CTV: Clinical target volume; DFS: Disease-free survival; FIGO: International Federation of Gynaecology and Obstetrics; GOG: Gynaecologic Oncology Group; GTV: Gross tumour volume; HR: Hazard ratio; ICBT: Intracavitary brachytherapy; IMRT: Intensity modulated radiation therapy; IRB: Institutional Review Board; MLNs: Metastatic lymph nodes; MVCT: Megavoltage computed tomography; OS: Overall survival; PCTV: Planning clinical target volume; PFS: Progression-free survival; PGTV: Planning gross tumour volume; SCC: Squamous cell carcinoma

\section{Acknowledgements}

None

\section{Funding}

This study was supported by the Ministry of Science and Technology of the People's Republic of China (grant number 2016YFC0105207).

\section{Availability of data and materials}

The datasets used and analysed during the current study are available from the corresponding author upon reasonable request.

\section{Authors' contributions}

WW was responsible for data collection and drafted the manuscript; $X L$ and QM participated in the design of the study. FZ and KH designed the study and revised the manuscript. All authors read and approved the manuscript.

Ethics approval and consent to participate

The Institutional Review Board (IRB) of Peking Union Medical College Hospital reviewed the protocol. This is retrospective study. The protocol is 
rational and scientific. The study is in accordance with ethical principles. The IRB thus approve the protocol.

The ethical committee process number is S-K450.

This is a retrospective study and written human subject consent was unnecessary.

\section{Consent for publication}

Not applicable

\section{Competing interests}

The authors declare that they have no competing interests.

\section{Publisher's Note}

Springer Nature remains neutral with regard to jurisdictional claims in published maps and institutional affiliations.

Received: 25 April 2018 Accepted: 4 December 2018

Published online: 17 December 2018

\section{References}

1. Siegel RL, Miller KD, Jemal A. Cancer statistics, 2018. CA Cancer J Clin. 2018; 68:7-30.

2. Watson M, Saraiya M, Benard V, Coughlin SS, Flowers L, et al. Burden of cervical cancer in the United States, 1998-2003. Cancer. 2008;113:2855-64.

3. Bray $F$, Loos AH, McCarron $P$, Weiderpass $E$, Arbyn $M$, et al. Trends in cervical squamous cell carcinoma incidence in 13 European countries: changing risk and the effects of screening. Cancer Epidemiol Biomark Prev. 2005; 14:677-86

4. Barnholtz-Sloan J, Patel N, Rollison D, Kortepeter K, MacKinnon J, et al. Incidence trends of invasive cervical cancer in the United States by combined race and ethnicity. Cancer Causes Control. 2009;20:1129-38.

5. Bray F, Carstensen B, Moller H, Zappa M, Zakelj MP, et al. Incidence trends of adenocarcinoma of the cervix in 13 European countries. Cancer Epidemiol Biomark Prev. 2005:14:2191-9.

6. Sasieni P, Castanon A, Cuzick J. Screening and adenocarcinoma of the cervix. Int J Cancer. 2009:125:525-9.

7. Rose PG, Java JJ, Whitney CW, Stehman FB, Lanciano R, et al. Locally advanced adenocarcinoma and adenosquamous carcinomas of the cervix compared to squamous cell carcinomas of the cervix in gynecologic oncology group trials of cisplatin-based chemoradiation. Gynecol Oncol. 2014:135:208-12.

8. Galic V, Herzog TJ, Lewin SN, Neugut Al, Burke WM, et al. Prognostic significance of adenocarcinoma histology in women with cervical cancer. Gynecol Oncol. 2012;125:287-91.

9. Wu SG, Sun JY, He ZY, Chen QH, Zhou J. Early-stage node negative cervical adenocarcinoma and squamous cell carcinoma show similar survival outcomes after hysterectomy: a population-based study. J Gynecol Oncol. 2017;28:e81.

10. Xie $X$, Song $K$, Cui $B$, Jiang J, Yang $X$, et al. A comparison of the prognosis between adenocarcinoma and squamous cell carcinoma in stage IB-IIA cervical cancer. Int J Clin Oncol. 2018:23:522-31.

11. Winer I, Alvarado-Cabrero I, Hassan O, Ahmed QF, Alosh B, et al. The prognostic significance of histologic type in early stage cervical cancer - a multi-institutional study. Gynecol Oncol. 2015;137:474-8.

12. Jung EJ, Byun JM, Kim YN, Lee KB, Sung MS, et al. Cervical adenocarcinoma has a poorer prognosis and a higher propensity for distant recurrence than squamous cell carcinoma. Int J Gynecol Cancer. 2017;27:1228-36.

13. Yamauchi M, Fukuda T, Wada T, Kawanishi M, Imai K, et al. Comparison of outcomes between squamous cell carcinoma and adenocarcinoma in patients with surgically treated stage $\mathrm{I}-\mathrm{Il}$ cervical cancer. Mol Clin Oncol. 2014:2:518-24

14. Mabuchi S, Okazawa M, Matsuo K, Kawano M, Suzuki O, et al. Impact of histological subtype on survival of patients with surgically-treated stage IA2-IIB cervical cancer: adenocarcinoma versus squamous cell carcinoma Gynecol Oncol. 2012;127:114-20.

15. Yokoi E, Mabuchi S, Takahashi R, Matsumoto Y, Kuroda H, et al. Impact of histological subtype on survival in patients with locally advanced cervical cancer that were treated with definitive radiotherapy: adenocarcinoma/ adenosquamous carcinoma versus squamous cell carcinoma. J Gynecol Oncol. 2017;28:e19.
16. Xiong Y, Liu J, Chen S, Zhou Q, Xu W, et al. Combination of external beam radiotherapy and californium (Cf)-252 neutron intracavity brachytherapy is more effective in control of cervical squamous cell carcinoma than that of cervical adenocarcinoma. Med Oncol. 2015;32:231.

17. Lee JY, Kim YT, Kim S, Lee B, Lim MC, et al. Prognosis of cervical cancer in the era of concurrent Chemoradiation from National Database in Korea: a comparison between squamous cell carcinoma and adenocarcinoma. PLoS One. 2015;10:e0144887.

18. Katanyoo K, Sanguanrungsirikul S, Manusirivithaya S. Comparison of treatment outcomes between squamous cell carcinoma and adenocarcinoma in locally advanced cervical cancer. Gynecol Oncol. 2012; 125:292-6.

19. Wang W, Liu X, Meng Q, Zhang F, Hu K. Prophylactic extended-field irradiation for patients with cervical cancer treated with concurrent Chemoradiotherapy: a propensity-score matching analysis. Int J Gynecol Cancer. 2018;28:1584-91

20. Wang W, Liu X, Meng Q, Zhang F, Hu K. Nomogram for predicting paraaortic lymph node metastases in patients with cervical cancer. Arch Gynecol Obstet. 2018:298:381-8.

21. Wang W, Hou X, Yan J, Shen J, Lian X, et al. Outcome and toxicity of radical radiotherapy or concurrent Chemoradiotherapy for elderly cervical cancer women. BMC Cancer. 2017:17:510.

22. Wang W, Zhang F, Hu K, Hou X. Image-guided, intensity-modulated radiation therapy in definitive radiotherapy for 1433 patients with cervical cancer. Gynecol Oncol. 2018;151:444-8.

23. Tang J, Tang Y, Yang J, Huang S. Chemoradiation and adjuvant chemotherapy in advanced cervical adenocarcinoma. Gynecol Oncol. 2012; 125:297-302.

24. Nagao S, Fujiwara K, Oda T, Ishikawa H, Koike H, et al. Combination chemotherapy of docetaxel and carboplatin in advanced or recurrent cervix cancer. A pilot study. Gynecol Oncol. 2005;96:805-9.

25. Curtin JP, Blessing JA, Webster KD, Rose PG, Mayer AR, et al. Paclitaxel, an active agent in nonsquamous carcinomas of the uterine cervix: a gynecologic oncology group study. J Clin Oncol. 2001;19:1275-8.

26. Huang YT, Wang CC, Tsai CS, Lai CH, Chang TC, et al. Long-term outcome and prognostic factors for adenocarcinoma/adenosquamous carcinoma of cervix after definitive radiotherapy. Int J Radiat Oncol Biol Phys. 2011;80:429-36.

Ready to submit your research? Choose BMC and benefit from:

- fast, convenient online submission

- thorough peer review by experienced researchers in your field

- rapid publication on acceptance

- support for research data, including large and complex data types

- gold Open Access which fosters wider collaboration and increased citations

- maximum visibility for your research: over $100 \mathrm{M}$ website views per year

At $\mathrm{BMC}$, research is always in progress.

Learn more biomedcentral.com/submissions 